\title{
Detection of Carotid Artery Stenosis: A Comparison between 2 Unenhanced MRAs and Dual-Source CTA
}

\author{
P. Lv, J. Lin, D. Guo, H. Liu, X. Tang, C. Fu, and J. Hu
}

\begin{abstract}
BACKGROUND AND PURPOSE: Dual-source CTA and black-blood MRA are recently developed techniques for evaluating carotid stenosis. The purpose of this study was to compare dual-source CTA with black-blood MRA and conventional TOF MRA in both detecting carotid stenosis by using DSA as a reference standard and demonstrating plaque morphology.
\end{abstract}

MATERIALS AND METHODS: Thirty patients with suspected carotid artery stenosis underwent unenhanced MRA by using black-blood and TOF MRA and dual-source CTA. Source images from unenhanced MRAs and dual-source CTA were reconstructed with MIP or curved planar reconstruction. The degree of carotid artery stenosis was measured, and plaque surface morphology at the stenosis was analyzed and compared among different techniques.

RESULTS: Good correlation was observed for measuring the degree of carotid stenosis among dual-source CTA, black-blood MRA, TOF MRA, and DSA. Sensitivity and specificity for detecting severe stenosis were $100 \%$ and $97 \%$ with dual-source CTA, $100 \%$ and $95 \%$ with black-blood MRA, and $79 \%$ and $95 \%$ with TOF MRA. None of the 3 technologies resulted in stenosis of $<50 \%$ being overestimated. Plaque surface irregularity or ulceration was more frequently detected with dual-source CTA and black-blood MRA than with TOF MRA and DSA.

CONCLUSIONS: This preliminary study shows that black-blood MRA is a promising technique, comparable with dual-source CTA and DSA, but better than TOF MRA, in the evaluation of carotid stenosis. Unlike dual-source CTA, black-blood MRA requires no intravenous contrast or radiation.

ABBREVIATIONS: BB MRA $=$ black-blood MRA; DSCTA $=$ dual-source CTA

C

arotid artery atherosclerosis is a major cause of ischemic cerebrovascular disease. ${ }^{1,2}$ Measurement of carotid stenosis and demonstration of plaque morphology are critical for the management of patients with carotid atherosclerosis. DSA is the current reference standard for evaluating carotid artery stenosis. The diagnostic role of DSA has largely been replaced, however, by noninvasive techniques such as sonography, CTA, and MRA.

Sonography has been the most commonly performed tech-

Received April 23, 2014; accepted after revision May 26

From the Department of Radiology (P.L., J.L., H.L.), Zhongshan Hospital, Shanghai Medical College of Fudan University and Shanghai Institute of Medical Imaging, Shanghai, China; Department of Vascular Surgery (D.G., X.T.), Zhongshan Hospital, Fudan University and Institute of Vascular Surgery, Shanghai, China; Siemens Shenzhen Magnetic Resonance (C.F.), Shenzhen, China; and Department of Cardiology (J.H.), Zhongshan Hospital, Fudan University, Shanghai, China.

Please address correspondence to Jiang Lin, MD, Department of Radiology, Zhongshan Hospital, Shanghai Medical College of Fudan University and Shanghai Institute of Medical Imaging, Fenglin Rd 180, Shanghai 200032, China; e-mail: lin.jiang@zshospital.sh.cn; and Daqiao Guo, MD, Department of Vascular Surgery, Zhongshan Hospital, Fudan University and Institute of Vascular Surgery, Fudan University, Fenglin Rd 180, Shanghai 200032, China; e-mail: guo.daqiao@zs-hospital.sh.cn

http://dx.doi.org/10.3174/ajnr.A4073 nique but may be restricted by its operator dependence and limited coverage. CTA is another widely used technique for the evaluation of carotid artery stenosis with high accuracy. ${ }^{3}$ Dual-source CTA (DSCTA) uses $2 \mathrm{x}$-ray sources and 2 detectors at the same time. With this technique, 2 images can be simultaneously acquired with different tube voltages; this feature has been shown to be an advantage for the evaluation of densely calcified carotid stenosis. ${ }^{4,5}$ Contrast-enhanced MRA has been established as an alternative for carotid imaging with a diagnostic accuracy similar to that of CTA. ${ }^{6,7}$ Both CTA and contrast-enhanced MRA use contrast media and are restricted in patients with impaired renal function, and CTA also requires ionizing radiation. As a result, unenhanced MRA without gadolinium is a desirable alternative, especially in patients with renal failure. Conventional TOF MRA has been widely used in clinical practice for carotid visualization, but it is limited by local reduction of signal intensity related to slow and turbulent flow and also prolonged imaging time. ${ }^{8}$ T2weighted black-blood MRA (BB MRA) is a newly developed technique showing potential in the evaluation of both the lumen and the wall of the carotid artery after optimal suppression of the 
signal from flowing blood. ${ }^{9,10}$ Few studies, to our knowledge however, have compared DSCTA with BB MRA and conventional TOF MRA in evaluating carotid stenosis.

The aim of this study was to prospectively and intraindividually compare these 2 unenhanced MRA methods with DSCTA in detecting carotid artery stenosis by using DSA as the standard of reference and in demonstrating plaque morphology.

\section{MATERIALS AND METHODS}

\section{Patients}

Between December 2013 and March 2014, 30 patients (mean age, $65 \pm 13$ years; 22 men and 8 women) suspected of having carotid atherosclerotic disease were prospectively enrolled in the study. All patients underwent DSCTA, TOF and BB MRA, and DSA sequentially within 2 weeks. The examinations were approved by the local medical ethics committee, and informed consent was obtained from each patient.

\section{DSCTA}

All examinations were performed on a dual-source CT system (Somatom Definition; Siemens, Erlangen, Germany). With a power injector, 50-70 $\mathrm{mL}$ (according to the patient's body weight) of nonionic iodinated contrast media (iopamidol, Iopamiron 370; Bracco, Milan, Italy) was injected into an antecubital vein at a flow rate of $5.0 \mathrm{~mL} / \mathrm{s}$ followed by a $25-\mathrm{mL}$ saline flush. CT was initiated by using a bolus-tracking technique at the level of the aortic arch with a trigger threshold of $100 \mathrm{HU}$. Acquisition parameters were $140 \mathrm{kV}$ and $80 \mathrm{mAs}_{\text {eff }}$ on tube A and $80 \mathrm{kV}$ and $234 \mathrm{mAs}_{\text {eff }}$ on tube B. Each detector was collimated to $32 \times 0.6$ $\mathrm{mm}$ with a flying focal spot, and a pitch of 0.65 was applied. Images were reconstructed with a dedicated D30 reconstruction algorithm. Section thickness and increment were 1.0 and $0.7 \mathrm{~mm}$, respectively. Two individual stacks of images for each detector ( 80 $\mathrm{kV}$ and $140 \mathrm{kV}$ images) and dual energy mixed images were reconstructed. The latter contained weighted information from both detectors with a weighting factor of 0.3 , thus approximating regular $120-\mathrm{kV}$ images.

The raw DSCTA images were transferred to a workstation with commercially available dual-energy postprocessing software (syngo Dual Energy; Siemens). Automatic bone removal was performed without further manual adjustments of the algorithm. The DSCTA images before and after bone removal were used for both reconstruction and diagnostic reading. The DSCTA images after bone removal were considered supplements in case calcified plaque affected the evaluation of carotid stenosis.

\section{MRA}

All examinations were performed on a 3T MR imaging system (Magnetom Verio; Siemens) using a head and neck coil. 3D TOF was acquired first with the following parameters: TR $=19 \mathrm{~ms}$; $\mathrm{TE}=3.6 \mathrm{~ms}$; flip angle $=18^{\circ} ; \mathrm{FOV}=240 \times 240 \mathrm{~mm}$; matrix $=$ $256 \times 256$; section thickness $=0.8 \mathrm{~mm}$; section number $=40$; mean acquisition time $=5: 33$ minutes. After that, 3D BB MRA was performed with a fat-saturated T2-sampling perfection with application-optimized contrast by using different flip angle evolution sequence in the coronal plane. Imaging parameters were as follows: $\mathrm{TR}=1800 \mathrm{~ms} ; \mathrm{TE}=168 \mathrm{~ms} ; \mathrm{FOV}=240 \times 240 \mathrm{~mm}$; matrix $=256 \times 256$; section thickness $=0.8 \mathrm{~mm}$; section number $=50$; mean acquisition time $=5: 47$ minutes.

\section{DSA}

DSA was performed via femoral artery catheterization by using the digital subtraction technique (Axiom Artis dTA; Siemens). Common carotid arteries were selectively catheterized. Images were obtained in anteroposterior, lateral, and 2 oblique projections for each catheterization.

\section{Image Analysis}

Commercially available 3D software (syngo, Siemens) was used to create MIP, MPR, and curved planar reconstructions from the raw DSCTA and MRA images.

An experienced vascular radiologist reviewed the image quality of DSCTA and MRA in 2 separate sessions. Image quality was evaluated on the basis of the following features: vascular attenuation or signal intensity, homogeneity of enhancement, and the presence of artifacts. It was scored on a 4-point scale as follows: 1 , excellent; 2, good; 3, moderate; and 4, poor visualization or nondiagnostic.

Each MRA and DSCTA image was assessed by another 2 experienced vascular radiologists (with 5 and 8 years of experience) independently in terms of stenosis and plaque morphology. A third interventional radiologist with 15 years of experience, who was unaware of the findings of the other examinations, assessed the stenosis and plaque surface on DSA. The patient's basic information was hidden, and all datasets were analyzed in random order. DSCTA, BB MRA, and TOF MRA images were evaluated separately with an interval of 1 week.

The degree of stenosis of each ICA was quantified on DSCTA, MRA, and DSA images on a similar plane. The diameter of the most severe stenosis was divided by the diameter of the ICA well beyond the bulb. The degree of stenosis was graded according to the NASCET criteria $^{11}$ : I (1\%-29\%), mild stenosis; II $(30 \%-$ $49 \%)$ and III (50\%-69\%), moderate stenosis; IV (70\%-99\%), severe stenosis; and V, occlusion. Plaque surface morphology was classified as regular, irregular, or ulcerated. ${ }^{12,13}$ Ulcer was seen as a crater penetrating into a plaque. A relatively smooth outpouching between 2 smooth narrowings that was most consistent with the expected position of the carotid wall would be regarded as a normal lumen and not ulceration.

\section{Statistical Assessment}

The relationship among DSCTA, BB MRA, TOF MRA, and DSA in grading stenosis was analyzed by using the Spearman rank correlation coefficient. For each unenhanced MRA and DSCTA, interobserver agreement in the evaluation of stenosis and plaque surface morphology was assessed by using the Cohen $\kappa$ test. Agreement was defined as mild $(\kappa>0.40-0.69)$, good $(\kappa>0.70-$ $0.89)$, or excellent $(\kappa>0.90-1.00)$.

\section{RESULTS}

In 30 patients, 55 stenoses at the carotid bifurcation were detected by DSA; 5 patients had unilateral carotid stenosis and 25 had bilateral carotid stenosis. The quality of DSCTA images was graded as either excellent (28 cases) or good ( 2 cases). No relevant 
Comparison of the degree of stenosis with CTA, BB MRA, TOF MRA, and DSA

\begin{tabular}{cccccc}
\hline \multirow{2}{*}{$\begin{array}{c}\text { Stenosis Degree on } \\
\text { DSCTA/BB MRA/TOF MRA }\end{array}$} & $\mathbf{1 \% - 2 9 \%}$ & $\mathbf{3 0 \% - 4 9 \%}$ & $\mathbf{5 0 \% - 6 9 \%}$ & $\mathbf{7 0 \% - 9 9 \%}$ & $\mathbf{1 0 0 \%}$ \\
\cline { 2 - 6 } Stenosis Degree on DSA \\
\hline 1\%-29\% & $23 / 23 / 23$ & $0 / 0 / 0$ & $0 / 0 / 0$ & $0 / 0 / 0$ & $0 / 0 / 0$ \\
$30 \%-49 \%$ & $0 / 0 / 0$ & $7 / 7 / 7$ & $0 / 0 / 0$ & $0 / 0 / 0$ & $0 / 0 / 0$ \\
$50 \%-69 \%$ & $0 / 0 / 0$ & $0 / 0 / 0$ & $7 / 6 / 6$ & $0 / 0 / 1$ & $0 / 0 / 0$ \\
$70 \%-99 \%$ & $0 / 0 / 0$ & $0 / 0 / 0$ & $1 / 2 / 2$ & $14 / 14 / 11$ & $0 / 0 / 0$ \\
$100 \%$ & $0 / 0 / 0$ & $0 / 0 / 0$ & $0 / 0 / 0$ & $0 / 0 / 2$ & $1 / 1 / 1$ \\
\hline
\end{tabular}
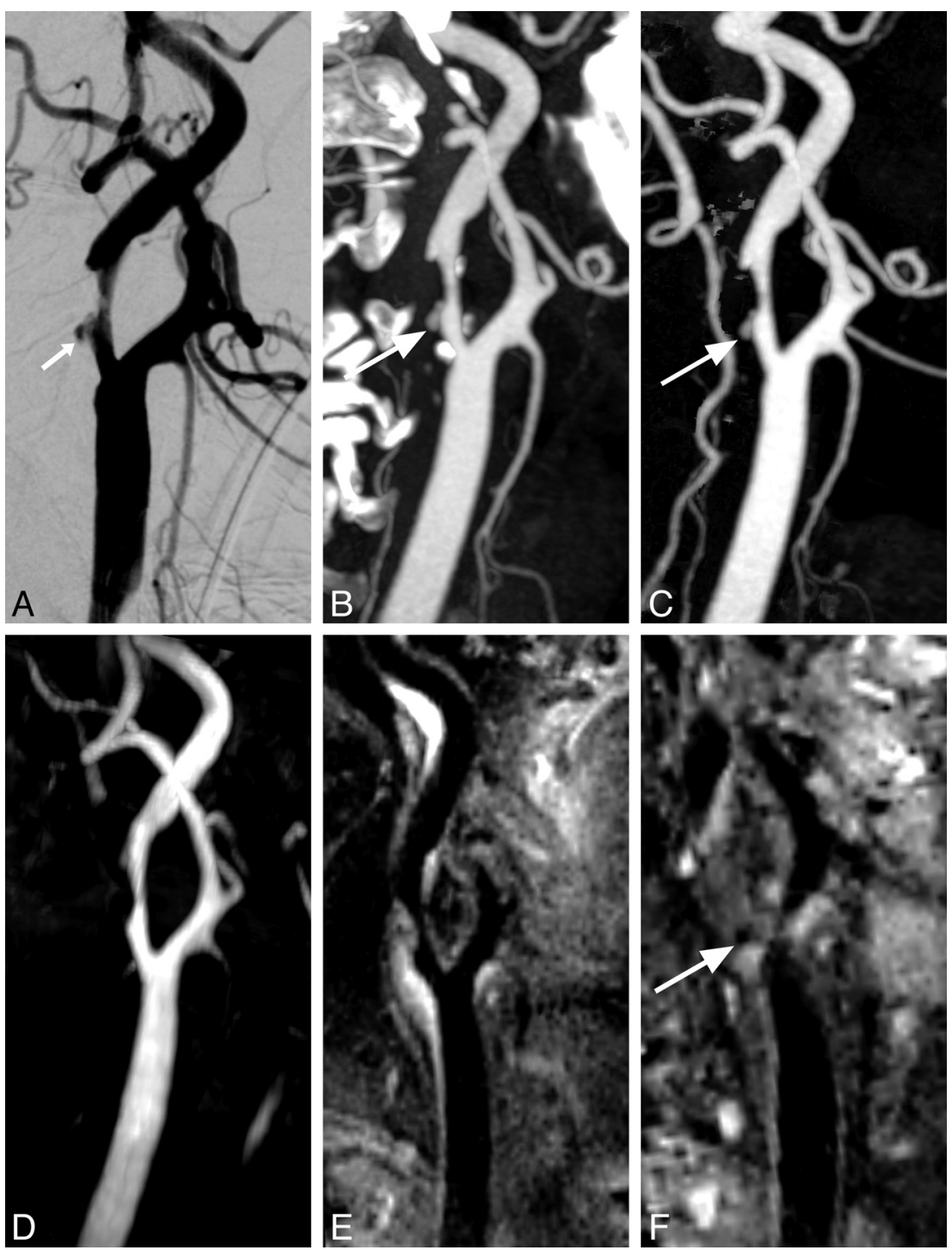

FIG 1. A, DSA depicts a moderate stenosis of the ICA. B, Dense calcification on standard DSCTA interferes with the display of the vascular lumen. C, DSCTA after removal of calcification and bone clearly demonstrates the stenosis. TOF MRA $(D)$ and BB MRA $(E)$ both accurately depict the degree of carotid stenosis. An ulcer (white arrows) is detected by all of the examinations except TOF MRA. F, MPR of BB MRA in a different perspective shows the ulcer.

motion artifacts diminished the quality of the DSCTA images. The TOF MRA was graded as either excellent (15 cases) or good (15 cases). The quality of BB MRA images was graded as excellent in 10 cases and good in 18 cases. In 2 cases, BB MRA was rated as poor visualization or nondiagnostic because of a limited signalto-noise ratio or motion artifacts. These 2 cases with unilateral carotid stenosis shown on DSA were excluded from further evaluation and comparison.
The Table summarizes the results of stenosis measured with DSCTA, BB MRA, and TOF MRA in comparison with DSA (Figs 1-3). For the measured carotid stenosis, good correlation was observed among these 3 techniques and DSA $\left(r^{2}=\right.$ $0.988,0.986,0.967$, respectively) (Fig 4 ). None of the 3 techniques resulted in the degree of stenosis $<50 \%$ being overestimated. Overestimation occurred in 1 case of moderate stenosis (50\%-69\%) with DSCTA and in 2 cases of moderate stenosis with both BB MRA and TOF MRA. TOF MRA misinterpreted severe stenosis (70\%-99\%) as occlusion in 2 cases.

Sensitivity and specificity for detecting severe stenosis was $100 \%$ and $97 \%$ with DSCTA, $100 \%$ and $95 \%$ with BB MRA, and $79 \%$ and $95 \%$ with TOF MRA. Interobserver agreement for the evaluation of the degree of stenosis was excellent for DSCTA $(\kappa=0.94)$ and good for BB MRA $(\kappa=0.81)$ and TOF MRA $(\kappa=0.85)$.

Plaque surface irregularity or ulcer was more frequently identified on DSCTA (18 irregularities and 7 ulcers) and BB MRA (19 irregularities and 6 ulcers) than on TOF MRA (15 irregularities and 3 ulcers) and DSA (16 irregularities and 4 ulcers). Three plaque ulcers all depicted by DSCTA, BB MRA, and DSA were not seen on TOF MRA (Fig 1). Interobserver agreement for the evaluation of plaque surface morphology was good for DSCTA $(\kappa=0.80)$, BB MRA $(\kappa=0.76)$, and TOF MRA $(\kappa=0.71)$.

\section{DISCUSSION}

Accurate evaluation of the degree of carotid stenosis is essential for guiding clinical treatment. Although DSA is still considered the criterion standard for the evaluation of carotid artery stenosis, there are some wellknown limitations, including its invasiveness, risk of neurologic complications, and the potential for variability in the quantification of stenosis, especially if limited projections are acquired.

Noninvasive vascular imaging techniques have gradually replaced DSA for the diagnosis of carotid artery stenosis. Previous studies have already proved the capabilities of both CTA and MRA to reliably determine the degree of carotid stenosis. ${ }^{6,14-17}$ In addition, studies have shown that CT and MR imaging may provide supplemental information about the composition and morphology of plaque. ${ }^{18-22}$

Due to its high spatial resolution and fast imaging, CTA has been widely used in the examination of the carotid artery. Identification of calcified plaque is another advantage of $\mathrm{CT}$, which is 

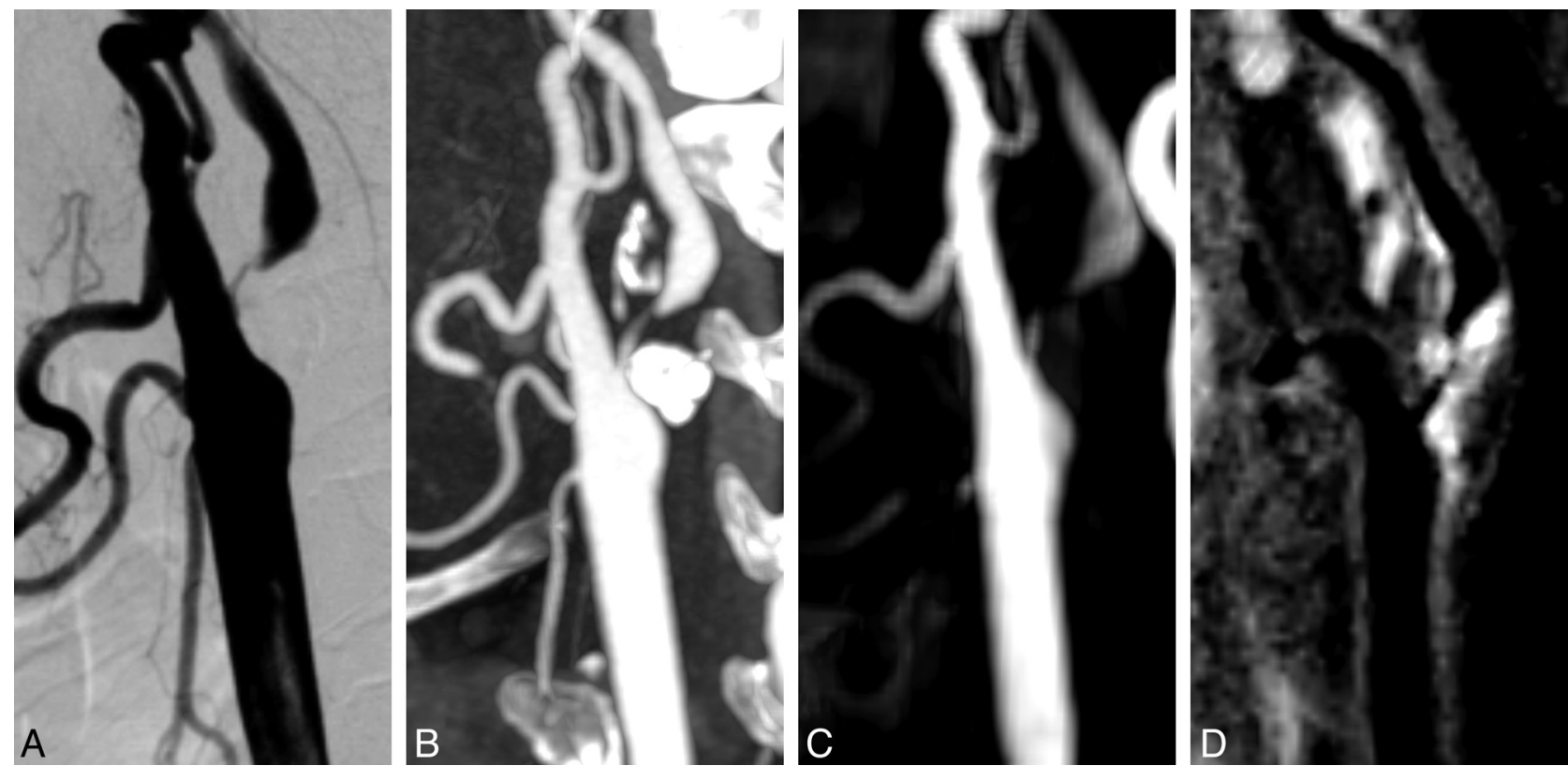

FIG 2. DSA $(A)$ and DSCTA $(B)$ depict a severe and irregular stenosis of the ICA. C, TOF MRA overestimates this stenosis as an occlusion because of local luminal nonvisualization. D, BB MRA clearly detects this severe stenosis with an irregular plaque surface.
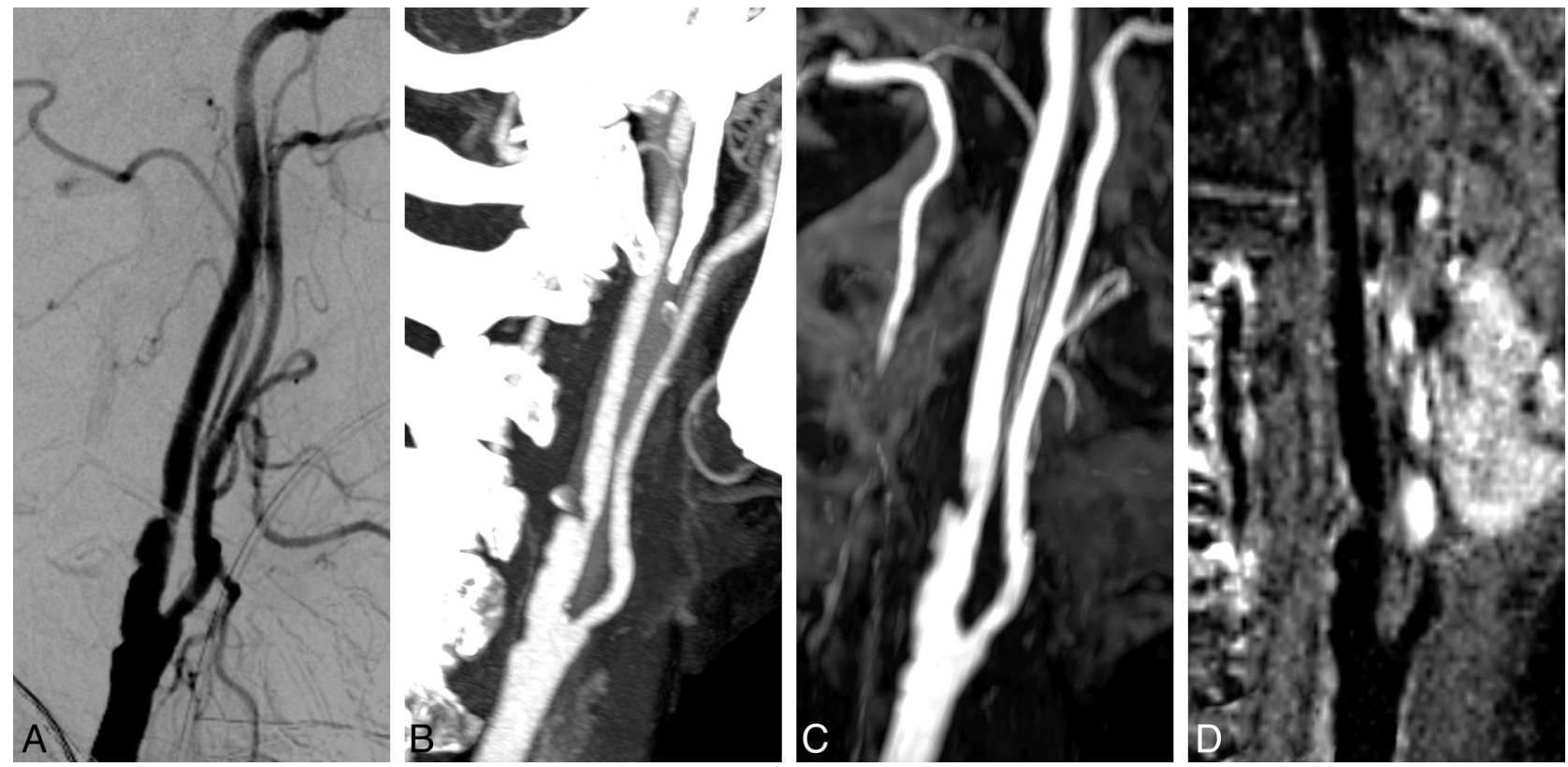

FIG 3. DSA (A), DSCTA (B), TOF MRA (C), and BB MRA (D) all depict a moderate and irregular stenosis at the ICA with close correlation.

helpful in the preinterventional assessment of carotid disease. However, in cases with severe calcification on the vessel wall, the demonstration of the lumen may be compromised; this compromise affects accurate evaluation of the degree of carotid stenosis. ${ }^{23,24}$

The application of conventional bone subtraction or automatic bone removal with DSCT greatly improves carotid assessment without overlay of calcified plaques. The dual-energy technique on DSCTA distinguishes contrast media from calcified plaques by exploiting the differences in attenuation of iodine and calcium at different $x$-ray energies. ${ }^{25}$ The radiation dose of a dualsource scan is only slightly higher than that of a normal singlesource scan. ${ }^{23}$ Compared with conventional subtraction, DSCTA reduces the radiation dose and avoids motion artifacts by sparing the unenhanced acquisition. ${ }^{23}$ With the dual-energy technique, we achieved the best image quality and accuracy of DSCTA in the demonstration of carotid stenosis. Sensitivity and specificity for detecting severe stenosis were $100 \%$ and $97 \%$ with DSCTA in our study, which was similar to that in the previous literature. ${ }^{23}$ Overestimation occurred in only 1 case because of excessive deletion of calcified plaque, which might be due to blooming artifacts and partial volume effects.

Although slightly inferior to CTA in the evaluation accuracy of carotid stenosis, contrast-enhanced MRA is also a robust technique. ${ }^{6}$ In recent years, however, nephrogenic systemic fibrosis in patients with renal failure associated with gadolinium chelates has

AJNR Am J Neuroradiol 35:2360-65 Dec 2014 www.ajnr.org

2363 

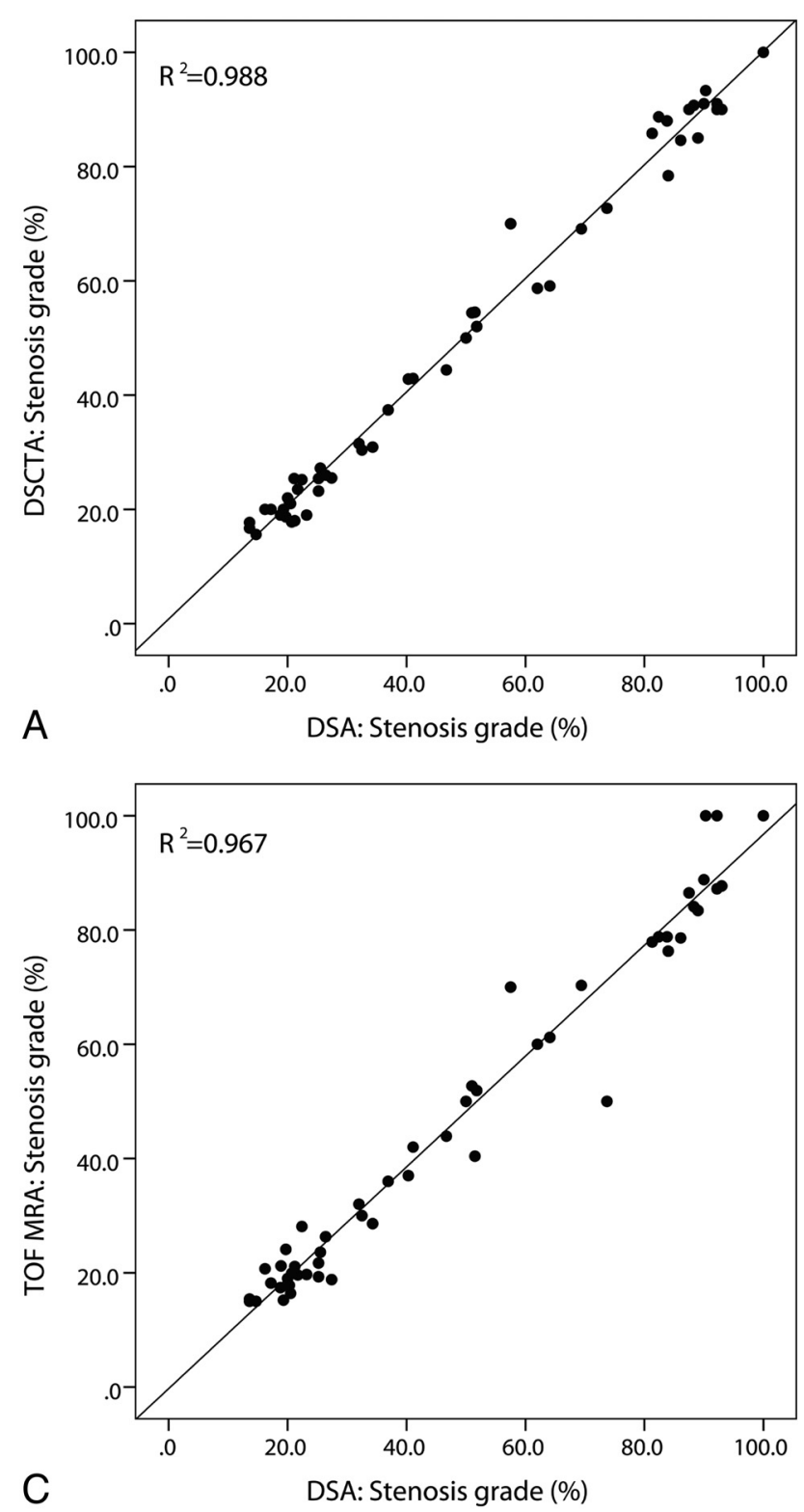

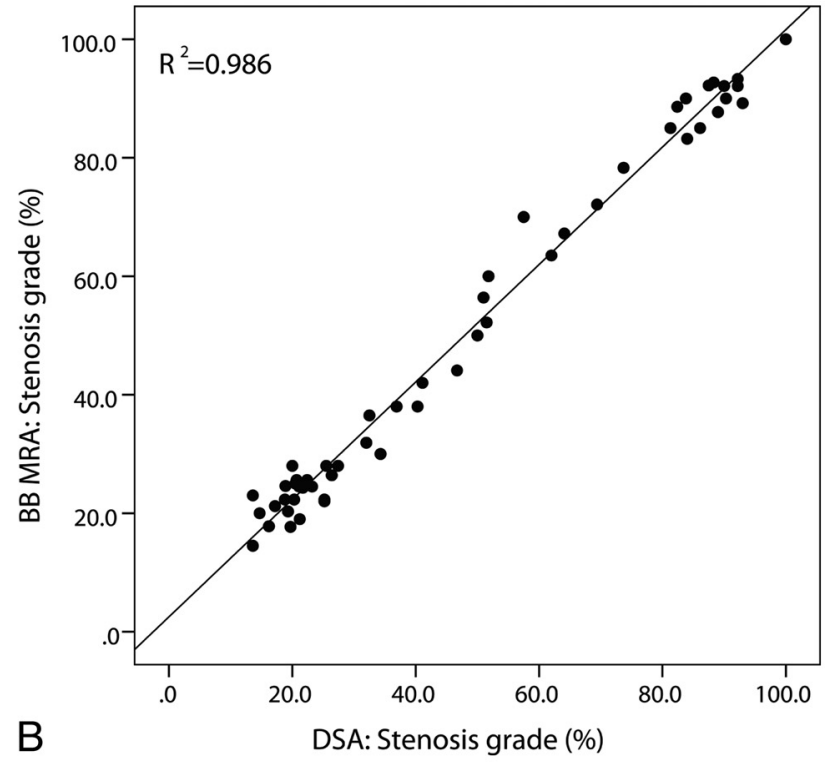

B

DSA: Stenosis grade $(\%)$

FIG 4. Linear regression among DSCTA, BB MRA, TOF MRA, and DSA for the stenosis measurement.

received increasing attention. ${ }^{26}$ As an alternative option, traditional TOF MRA is still widely in use despite signal loss resulting from slow and turbulent flow at the carotid bifurcation region. ${ }^{8}$ In this study, overestimation occurred on TOF MRA in 2 cases with moderate stenosis and in 2 cases with severe stenosis.

Black-blood MR imaging has been used to define carotid plaque composition and morphology. ${ }^{20,27}$ In recent years, it is being refined to visualize and quantify the lumen of the carotid artery. ${ }^{10,28}$ By selectively suppressing the signals coming from the artery lumen, BB MRA can better delineate the structure of the arterial wall and detect the degree of carotid stenosis. 3D sampling perfection with the application of optimized contrast by using different flip angle evolution is a recently developed T2-weighted MR imaging technique with high spatial resolution. Good T2weighted images can be obtained by using different flip angles, which enable a low specific absorption rate value. Use of a parallel acquisition technique makes it possible to obtain thin-section images in a reasonable acquisition time. ${ }^{29}$ One previous study showed that 3D BB MRA could allow accurate measurement of carotid stenosis in comparison with contrast-enhanced MRA. ${ }^{10}$ Our study showed that its sensitivity and specificity for the detection of severe carotid stenosis were comparable with DSCTA but better than TOF, though the image quality was slightly inferior to that of DSCTA. Overestimation occurred in 2 cases of moderate stenosis, which was due to heterogeneous signals from the lumen.

Detecting plaque morphology is also important. Ulceration and irregularity of carotid plaque are strong predictors of overall carotid plaque instability. ${ }^{12}$ Furthermore, they have been shown to predict the risk of future stroke and to be associated with acute coronary events. ${ }^{30}$ Because of the limited projection angles, DSA has limitations in detecting irregular plaque or small ulcers.

In our study, irregularities of plaques and ulcers were more frequently observed on DSCTA and BB MRA because both allowed better visualization of the plaque through multiple perspectives. TOF MRA, however, was insensitive to the surface ulcer 
of the carotid plaque, which might be due to the local loss of signal intensity as mentioned above.

This study has several limitations. First, the sample size was relatively small, and further studies are warranted to verify the accuracy of BB MRA in evaluating carotid stenosis. Second, no surgical and histologic confirmation was available for plaque morphology in our study. We assumed that the number of ulcers detected in this study is likely be underestimated because small ulcers might not be obvious on all images or appeared as plaque irregularities. Finally, the acquisition time of BB MRA was still long, and further technical optimization of BB MRA was still needed because 2 cases were excluded from our study owing to motion artifacts or reduced signal-to-noise ratio.

\section{CONCLUSIONS}

BB MRA is a promising technique that is comparable with DSCTA and DSA in the evaluation of carotid stenosis. In addition, BB MRA and DSCTA are better than TOF MRA in demonstrating stenosis and plaque morphology. The clinical application of BB MRA may be valuable, given that it does not use ionizing radiation and contrast medium.

\section{REFERENCES}

1. Inzitari D, Eliasziw $\mathrm{M}$, Gates $\mathrm{P}$, et al. The causes and risk of stroke in patients with asymptomatic internal-carotid-artery stenosis: North American Symptomatic Carotid Endarterectomy Trial Collaborators. N Engl J Med 2000;342:1693-700

2. Barnett HJ, Gunton RW, Eliasziw M, et al. Causes and severity of ischemic stroke in patients with internal carotid artery stenosis. JAMA 2000;283:1429-36

3. Koelemay MJ, Nederkoorn PJ, Reitsma JB, et al. Systematic review of computed tomographic angiography for assessment of carotid artery disease. Stroke 2004;35:2306-12

4. Korn A, Bender B, Thomas C, et al. Dual energy CTA of the carotid bifurcation: advantage of plaque subtraction for assessment of grade of the stenosis and morphology. Eur J Radiol 2011;80:e120-25

5. Vlahos I, Chung R, Nair A, et al. Dual-energy CT: vascular applications. AJR Am J Roentgenol 2012;199:S87-97

6. Randoux B, Marro B, Koskas F, et al. Carotid artery stenosis: prospective comparison of CT, three-dimensional gadolinium-enhanced MR, and conventional angiography. Radiology 2001;220: $179-85$

7. Villablanca JP, Nael K, Habibi R, et al. 3 T contrast-enhanced magnetic resonance angiography for evaluation of the intracranial arteries: comparison with time-of-flight magnetic resonance angiography and multislice computed tomography angiography. Invest Radiol 2006;41:799-805

8. Miyazaki M, Lee VS. Nonenhanced MR angiography. Radiology 2008;248:20-43

9. Balu N, Yarnykh VL, Chu B, et al. Carotid plaque assessment using fast 3D isotropic resolution black-blood MRI. Magn Reson Med 2011;65:627-37

10. Mihai G, Winner MW, Raman SV, et al. Assessment of carotid stenosis using three-dimensional T2-weighted dark blood imaging: initial experience. J Magn Reson Imaging 2012;35:449-55

11. North American Symptomatic Carotid Endarterectomy Trial: methods, patient characteristics and progress. Stroke 1991;22: 711-20

12. Lovett JK, Gallagher PJ, Hands LJ, et al. Histological correlates of carotid plaque surface morphology on lumen contrast imaging. Circulation 2004;110:2190-97

13. Streifler JY, Eliasziw M, Fox AJ, et al. Angiographic detection of carotid plaque ulceration: comparison with surgical observations in a multicenter study: North American Symptomatic Carotid Endarterectomy Trial. Stroke 1994;25:1130-32

14. Remonda L, Senn P, Barth A, et al. Contrast-enhanced 3D MR angiography of the carotid artery: comparison with conventional digital subtraction angiography. AJNR Am J Neuroradiol 2002;23: 213-19

15. Josephson SA, Bryant SO, Mak HK, et al. Evaluation of carotid stenosis using CT angiography in the initial evaluation of stroke and TIA. Neurology 2004;63:457-60

16. Cumming MJ, Morrow IM. Carotid artery stenosis: a prospective comparison of CT angiography and conventional angiography. AJR Am J Roentgenol 1994;163:517-23

17. Huston JR, Fain SB, Wald JT, et al. Carotid artery: elliptic centric contrast-enhanced MR angiography compared with conventional angiography. Radiology 2001;218:138-43

18. Wintermark M, Jawadi SS, Rapp JH, et al. High-resolution CT imaging of carotid artery atherosclerotic plaques. AJNR Am J Neuroradiol 2008;29:875-82

19. Cormode DP, Roessl E, Thran A, et al. Atherosclerotic plaque composition: analysis with multicolor CT and targeted gold nanoparticles. Radiology 2010;256:774-82

20. Saam T, Hatsukami TS, Takaya N, et al. The vulnerable, or high-risk, atherosclerotic plaque: noninvasive MR imaging for characterization and assessment. Radiology 2007;244:64-77

21. Qiao Y, Etesami M, Astor BC, et al. Carotid plaque neovascularization and hemorrhage detected by MR imaging are associated with recent cerebrovascular ischemic events. AJNR Am J Neuroradiol 2012;33:755-60

22. van den Bouwhuijsen QJ, Vernooij MW, Hofman A, et al. Determinants of magnetic resonance imaging detected carotid plaque components: the Rotterdam Study. Eur Heart J 2012;33:221-29

23. Uotani K, Watanabe Y, Higashi M, et al. Dual-energy CT head bone and hard plaque removal for quantification of calcified carotid stenosis: utility and comparison with digital subtraction angiography. Eur Radiol 2009;19:2060-65

24. Deng K, Liu C, Ma R, et al. Clinical evaluation of dual-energy bone removal in CT angiography of the head and neck: comparison with conventional bone-subtraction CT angiography. Clin Radiol 2009; 64:534-41

25. Johnson TR, Krauss B, Sedlmair M, et al. Material differentiation by dual energy CT: initial experience. Eur Radiol 2007;17:1510-17

26. Thomsen HS. Nephrogenic systemic fibrosis: a serious late adverse reaction to gadodiamide. Eur Radiol 2006;16:2619-21

27. Kerwin WS, Hatsukami T, Yuan C, et al. MRI of carotid atherosclerosis. AJR Am J Roentgenol 2013;200:W304-13

28. Kramer H, Runge VM, Morelli JN, et al. Magnetic resonance angiography of the carotid arteries: comparison of unenhanced and contrast enhanced techniques. Eur Radiol 2011;21:1667-76

29. Haystead CM, Dale BM, Merkle EM. N/2 ghosting artifacts: elimination at 3.0-T MR cholangiography with SPACE pulse sequence. Radiology 2008;246:589-95

30. Rothwell PM, Villagra R, Gibson R, et al. Evidence of a chronic systemic cause of instability of atherosclerotic plaques. Lancet 2000; 355:19-24 